\title{
INTERVAL ESTIMATION OF KUMARASWAMY PARAMETERS BASED ON PROGRESSIVELY TYPE II CENSORED SAMPLE AND RECORD VALUES
}

\author{
HANIEH PANAHI
}

Received 08 June, 2018

\begin{abstract}
In this paper, interval estimation for the parameters of the Kumaraswamy distribution is studied based on progressively censoring scheme and the record values. Some pivotal quantities and Theorems are proposed to construct the exact confidence interval for the one-shape parameter and the joint confidence region for the two-shape parameters. Simulation study is performed to investigate the coverage probabilities of the proposed confidence under progressively censored sample and upper record data. Finally, the proposed intervals and regions have been studied based on two real data sets as the illustrative examples. The first data set contains the strength characterization of brittle material and the second data set consists of the daily average wind speeds.
\end{abstract}

2010 Mathematics Subject Classification: 62N02; 62P30

Keywords: brittle material, generalized spacing, joint confidence region, Kumaraswamy distribution, progressively censoring, upper record values, wind speed

\section{INTRODUCTION}

The Kumaraswamy (KU) distribution was introduced in the literature by Kumaraswamy ([11]) in connection with a problem in hydrology. This distribution has many similarities to the Beta distribution but it has a number of advantages. The KU distribution can be better suited than the Beta distribution for computation-intensive activities like simulation modeling and the estimation of models by simulation-based methods [15]. It has an invertible closed form cumulative distribution function which makes it more useful than the Beta distribution. Due to its practicality, it can be used for many applications, including atmospheric temperatures, wind speeds, scores obtained on a test, daily rainfall, daily stream flow, hydrological data and etc. The probability density function and the cumulative distribution function of KU distribution are given by:

$$
f(x ; \alpha, \beta)=\alpha \beta x^{\beta-1}\left(1-x^{\beta}\right)^{\alpha-1} ; 0<x<1, \alpha>0, \beta>0,
$$


and

$$
F(x ; \alpha, \beta)=1-\left(1-x^{\beta}\right)^{\alpha} ; 0<x<1
$$

respectively. The corresponding quantile function is:

$$
Q(p ; \alpha, \beta)=\left(1-\left(1-p^{1 / \alpha}\right)\right)^{1 / \beta} ; \quad 0<p<1
$$

The KU distribution is unimodal, uniantimodal, increasing, decreasing or constant depending (in the same way as the Beta distribution) on the values of its parameters [10]. If the parameters are greater than one, the density function is unimodal. It is uniantimodal if two parameters are less than one. The density function increases (decreases) for $\beta>1$ and $\alpha \leq 1$ ( $\beta \leq 1$ and $\alpha>1$ ) and finally, it is constant for $\alpha=\beta=1$. In Figure 1, we have displayed probability density function of the KU distribution for some arbitrarily selected parameter values.

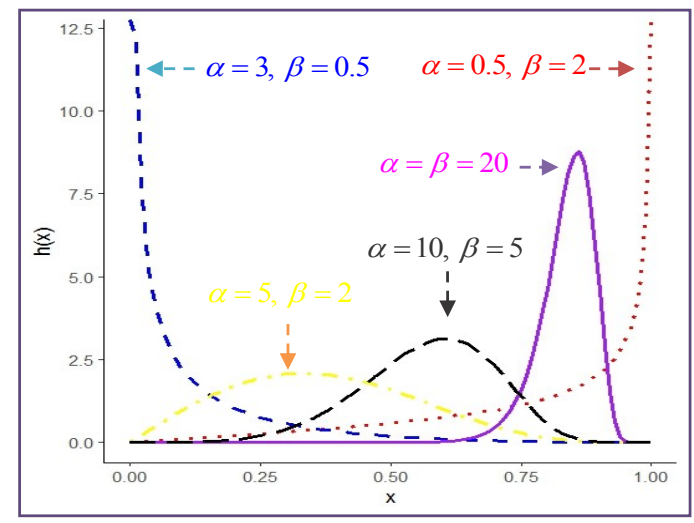

FIGURE 1 . The probability density function for different values of $\alpha$ and $\beta$.

Several author studied different aspects of the KU distribution. For example the distribution of order statistics from the KU model has been introduced and studied by [8]. The different estimation methods for the unknown parameters of the KU distribution have been considered by [6]. The point estimations for this distribution under complete and record samples have been studied by [13,16]. Furthermore, for more details about the other extensions of this distribution, see [7,22,25]. Although several articles have been done on the KU distribution but we have not come across any article about the exact confidence interval (ECI) and the joint confidence region (JCR) under progressively censored sample and record data. The main aim of this paper is two fold. First we try to introduce the ECI and JCR for parameter(s) on the basis of progressively Type II (PII) censoring using the appropriate pivotal quantities. Censoring is common in life tests because of time limits and other restrictions on data. Two of the commonly used censoring schemes are Type I and Type II censoring schemes. 
A mixture of Type I and Type II censoring schemes is known as the hybrid censoring scheme (see, [4,12,19] and [18]). These censoring schemes do not allow for removal of units at points other than the terminal point of the experiment. To overcome this problem, progressively censoring scheme which enables us to use live units, removed early, in other tests has been introduced. The PII censoring scheme can be implemented as follows: Suppose $n$ units are placed on a life test and the experimenter decides beforehand the quantity $m$, the number of units to be failed. Now at the time of the first failure, $R_{1}$ of the remaining $n-1$ surviving units are randomly removed from the experiment. Continuing on, at the time of the second failure, $R_{2}$ of the remaining $n-2-R_{1}$ units are randomly with-drawn from the experiment. Finally, at the time of the $m^{\text {th }}$ failure, all the remaining $R_{m}=n-m-R_{1}-R_{2}-\ldots-R_{m-1}$ surviving units are removed from the experiment. The $R_{i}^{\prime} s$ are fixed prior to the study. Some of the work on PII censoring was conducted by $[14,20]$ and [1].

Moreover, the study of record values and associated statistics are important in different multiple real life situations such as manufacturing industry, hydrology, seismology, sporting and stock market analysis. Since then, numerous papers on record values and their distributional properties have been focus of investigation for many authors, see for example,[5, 9,23] and [21]. A record values can be explained as follows. Suppose that $\left\{X_{i} ; i \geq 1\right\}$ denote a sequence of independent and identically distributed random variables with probability density function $f(x, \theta)$. Then, an observation $X_{i}$ is called an upper record if its value is greater than all preceding observations, that is, if $X_{i}>X_{j}$ for every $i>j$. The lower record value can also be defined in a similar manner. Also, the sequence $\left\{X_{U(n)} ; n \geq 1\right\}$ is a sequence of upper record statistics, where

$$
U(n)=\min \left\{i: i>U(n-1), X_{i}>X_{U(n-1)}\right\} ; n \geq 2, U(1)=1 .
$$

So, the second aim of this paper is to construct the ECI and JCR based on upper record values using the two Theorems. The rest of the paper is organized as follows. In Section 2, the ECI for the parameter $\beta$ and JCR for $(\alpha, \beta)$ are obtained by using some pivotal quantities. Based on record values, the exact and joint confidence are presented in Section 3. Section 4 deals with Monte Carlo simulation study. For illustration, two real data sets are discussed in Section 5 and finally we conclude the paper in Section 6.

\section{The ECI ANd JCR based on Progressively Censored SAMPle}

This section deals with constructing the ECI and JCR under PII censoring scheme. Let $X_{1: m: n}<X_{2: m: n}<\ldots<X_{m: m: n}$ denote the PII censored order statistics from KU distribution with censoring scheme $R=\left(R_{1}, \ldots, R_{m}\right)$. Let, $Y_{i}=-\log \left(1-X_{i: m: n}^{\beta}\right)^{\alpha}$; $i=1,2, \ldots, m$, it can be observed that $Y_{1: m: n}<\ldots<Y_{m: m: n}$ are the PII censored order statistics from a standard exponential distribution. Now, we consider the following 
transformation

$$
\begin{gathered}
\Delta_{1}=n Y_{1: m: n}, \\
\Delta_{2}=\left(n-R_{1}-1\right)\left(Y_{2: m: n}-Y_{1: m: n}\right), \\
\Delta_{3}=\left(n-R_{1}-R_{2}-2\right)\left(Y_{3: m: n}-Y_{2: m: n}\right), \\
\vdots \\
\Delta_{m}=\left(n-R_{1}-\ldots-R_{m-1}-m+1\right)\left(Y_{m: m: n}-Y_{m-1: m: n}\right) .
\end{gathered}
$$

It is observed that $\Delta_{1}, \Delta_{2}, \ldots, \Delta_{m}$ are independent and identically distributed as an exponential distribution with mean 1 . We introduce the following quantities:

$$
v_{1}=2 \Delta_{1}=2 n Y_{1: m: n}=-2 n \log \left(1-X_{1: m: n}^{\beta}\right)^{\alpha} \sim \chi^{2}(2),
$$

and

$$
v_{2}=2\left(\Delta_{2}+\Delta_{3}+\ldots+\Delta_{m}\right)=2 \sum_{i=1}^{m}\left(R_{i}+1\right)\left(Y_{i: m: n}-Y_{1: m: n}\right) \sim \chi^{2}(2 m-2) .
$$

We can find that $v_{1}$ and $v_{2}$ are independent. Now, suppose that

$$
W_{1}=v_{1}+v_{2}=2 \sum_{i=1}^{m}\left(R_{i}+1\right) Y_{i}=-2 \sum_{i=1}^{m}\left(R_{i}+1\right) \log \left(1-X_{i: m: n}^{\beta}\right)^{\alpha} \sim \chi^{2}(2 m),
$$

and

$$
\begin{aligned}
W_{2}= & \frac{2 v_{2}}{2(m-1) v_{1}} \\
= & \frac{\sum_{i=1}^{m}\left(R_{i}+1\right)\left(\log \left(1-X_{i: m: n}^{\beta}\right)^{\alpha}-\log \left(1-X_{1: m: n}^{\beta}\right)^{\alpha}\right)}{n(m-1) \log \left(1-X_{1: m: n}^{\beta}\right)^{\alpha}} \sim F(2 m-2,2) .
\end{aligned}
$$

It is also clear that $W_{1}$ and $W_{2}$ are independent. The following Lemma and Theorems enable us to construct the ECI and JCR for the PII censored sample.

Lemma 1. For a PII censored sample $X_{1: m: n}, X_{2: m: n}, \ldots, X_{m: m: n}$, the

$$
W_{2}(\beta ; \text { dat } a)=\frac{\sum_{i=1}^{m}\left(R_{i}+1\right)\left(\frac{\log \left(1-X_{i m: n}^{\beta}\right)}{\log \left(1-X_{1: m: n}^{\beta}\right)}-1\right)}{n(m-1)}
$$

is a strictly increasing function of $\beta$, where $\beta>0$. Furthermore, there is a unique solution for the given equation $W_{2}(\beta ;$ data $)=t$.

Proof. The proof follows using similar arguments of [24] and therefore it is omitted. 
Theorem 1. Suppose that $X_{i: m: n} ; i=1, \ldots, m$ are PII censored order statistics from $K U$ distribution with censoring scheme $R=\left(R_{1}, \ldots, R_{m}\right)$. Then, based on the pivotal quantities $W_{1}$ and $W_{2}$, a $100(1-\gamma) \%$ ECI for $\beta$ can be written as:

$$
\begin{aligned}
\varphi\left(X_{1: m: n}, X_{2: m: n}, \ldots, X_{m: m: n}, R_{1}, \ldots, R_{m}, F_{1-\gamma / 2}(2 m-2,2)\right) & <\beta \\
& <\varphi\left(X_{1: m: n}, X_{2: m: n}, \ldots, X_{m: m: n}, R_{1}, \ldots, R_{m}, F_{\gamma / 2}(2 m-2,2)\right)
\end{aligned}
$$

where, $\varphi\left(X_{1: m: n}, X_{2: m: n}, \ldots, X_{m: m: n}, R_{1}, \ldots, R_{m}, t\right)$ is the solution of $\beta$ for the following equation

$$
\frac{\sum_{i=1}^{m}\left(R_{i}+1\right)\left(\frac{\log \left(1-X_{i: m: n}^{\beta}\right)}{\log \left(1-X_{1: m: n}^{\beta}\right)}-1\right)}{n(m-1)}=t .
$$

Proof of Theorem 1. From (2.2), we know that the quantity

$$
W_{2}(\beta ; \text { data })=\frac{\sum_{i=1}^{m}\left(R_{i}+1\right)\left(\frac{\log \left(1-X_{i m: n}^{\beta}\right)}{\log \left(1-X_{1: m: n}^{\beta}\right)}-1\right)}{n(m-1)},
$$

has a $F$ distribution with $2 m-2$ and degrees of freedom. So, using the Lemma, we can conclude that $W_{2}(\beta ;$ data $)=t$, has a unique solution for any $\beta>0$ ([17]). Hence the event

$$
t_{1}<\frac{\sum_{i=1}^{m}\left(R_{i}+1\right)\left(\frac{\log \left(1-X_{i: m: n}^{\beta}\right)}{\log \left(1-X_{1: m: n}^{\beta}\right)}-1\right)}{n(m-1)}<t_{2},
$$

is equivalent to

$$
\begin{aligned}
\varphi\left(X_{1: m: n}, X_{2: m: n}, \ldots, X_{m: m: n}, R_{1}, \ldots, R_{m}, F_{1-\gamma / 2}(2 m-2,2)\right)<\beta \\
\quad<\varphi\left(X_{1: m: n}, X_{2: m: n}, \ldots, X_{m: m: n}, R_{1}, \ldots, R_{m}, F_{\gamma / 2}(2 m-2,2)\right)
\end{aligned}
$$

Now we want to obtain the JCR for $(\alpha, \beta)$ using the following Theorem.

Theorem 2. Suppose that $X_{i: m: n} ; i=1, \ldots, m$ are PII censored order statistics from $K U$ distribution with censoring scheme $R=\left(R_{1}, \ldots, R_{m}\right)$.Then, the following inequalities determine a $100(1-\gamma) \%$ JCR for $(\alpha, \beta)$.

$$
\begin{gathered}
\beta_{L}<\beta<\beta_{U} \\
-\frac{\chi_{(1+\sqrt{1-\gamma}) / 2}^{2}(2 m)}{2 \sum_{i=1}^{m}\left(R_{i}+1\right) \log \left(1-X_{i: m: n}^{\beta}\right)}<\alpha<-\frac{\chi_{(1-\sqrt{1-\gamma}) / 2}^{2}(2 m)}{2 \sum_{i=1}^{m}\left(R_{i}+1\right) \log \left(1-X_{i: m: n}^{\beta}\right)},
\end{gathered}
$$

where,

$$
\beta_{L}=\varphi\left(X_{1: m: n}, X_{2: m: n}, \ldots, X_{m: m: n}, R_{1}, \ldots, R_{m}, F_{(1+\sqrt{1-\gamma}) / 2}(2 m-2,2)\right),
$$


and

$$
\beta_{U}=\varphi\left(X_{1: m: n}, X_{2: m: n}, \ldots, X_{m: m: n}, R_{1}, \ldots, R_{m}, F_{(1-\sqrt{1-\gamma}) / 2}(2 m-2,2)\right) .
$$

Also, $\varphi\left(X_{1: m: n}, X_{2: m: n}, \ldots, X_{m: m: n}, R_{1}, \ldots, R_{m}, t\right)$ is introduced in Theorem 1.

Proof of Theorem 2. The $W_{1}=-2 \sum_{i=1}^{m}\left(R_{i}+1\right) \log \left(1-X_{i: m: n}^{\beta}\right)^{\alpha}$ has a $\chi^{2}$ distribution with $2 m$ degrees of freedom, and it is independent of $W_{2} \sim F(2 m-2,2)$. Therefore,

$$
P\left(\chi_{(1+\sqrt{1-\gamma}) / 2}^{2}(2 m)<W_{1}<\chi_{(1-\sqrt{1-\gamma}) / 2}^{2}(2 m)\right)=\sqrt{1-\gamma}
$$

and

$$
P\left(F_{(1+\sqrt{1-\gamma}) / 2}(2 m-2,2)<W_{2}<F_{(1-\sqrt{1-\gamma}) / 2}(2 m-2,2)\right)=\sqrt{1-\gamma} .
$$

So, we can write

$$
\begin{aligned}
1-\gamma= & \sqrt{1-\gamma} \times \sqrt{1-\gamma} \\
= & P\left(F_{(1+\sqrt{1-\gamma}) / 2}(2 m-2,2)<W_{2}<F_{(1-\sqrt{1-\gamma}) / 2}(2 m-2,2)\right) \\
& \times P\left(\chi_{(1+\sqrt{1-\gamma}) / 2}^{2}(2 m)<W_{1}<\chi_{(1-\sqrt{1-\gamma}) / 2}^{2}(2 m)\right) \\
= & P\left(F_{(1+\sqrt{1-\gamma}) / 2}(2 m-2,2)<W_{2}<F_{(1-\sqrt{1-\gamma}) / 2}(2 m-2,2),\right. \\
& \left.\chi_{(1+\sqrt{1-\gamma}) / 2}^{2}(2 m)<W_{1}<\chi_{(1-\sqrt{1-\gamma}) / 2}^{2}(2 m)\right) \\
= & P\left(\beta_{L}<\beta<\beta_{U},-\frac{\chi_{(1+\sqrt{1-\gamma}) / 2}^{2}(2 m)}{\kappa_{i}}<\alpha<-\frac{\chi_{(1-\sqrt{1-\gamma}) / 2}^{2}(2 m)}{\kappa_{i}}\right),
\end{aligned}
$$

where, $\kappa_{i}=2 \sum_{i=1}^{m}\left(R_{i}+1\right) \log \left(1-X_{i: m: n}^{\beta}\right)$. This completes the proof.

\section{THE ECI AND JCR UNDER THE UPPER RECORD VALUES}

In this section based on the upper record values, the exact confidence interval for $\beta$ and the JCR for $(\alpha, \beta)$ are introduced. Suppose that $X_{U(1)}<X_{U(2)}<\ldots<X_{U(m)}$ is the first $m$ upper record values from the KU distribution. Similarly to the previous section, let

$$
Y_{i}=-\log \left(1-X_{U(i)}^{\beta}\right)^{\alpha} ; i=1, \ldots, m .
$$

It can be seen that $Y_{1}<\ldots<Y_{m}$ are the first $m$ upper record values from an exponential distribution with mean 1 . We know that the following generalized spacings are independent and identically distributed as a standard exponential distribution [2].

$$
\begin{gathered}
Y_{1}^{*}=Y_{1}=-\log \left(1-X_{U(1)}^{\beta}\right)^{\alpha} \\
Y_{2}^{*}=Y_{2}-Y_{1}=\log \left(1-X_{U(2)}^{\beta}\right)^{\alpha}-\log \left(1-X_{U(1)}^{\beta}\right)^{\alpha}
\end{gathered}
$$




$$
Y_{m}^{*}=Y_{m}-Y_{m-1}=\log \left(1-X_{U(m)}^{\beta}\right)^{\alpha}-\log \left(1-X_{U(m-1)}^{\beta}\right)^{\alpha}
$$

Hence, $W_{1}=2 Y_{1}^{*}=2 Y_{1}$ and $W_{2}=2 \sum_{i=2}^{m} Y_{i}^{*}=2\left(Y_{m}-Y_{1}\right)$, have the chi-squared distribution with $2 m$ and $2 m-2$ degrees of freedom respectively. In order to construct the ECI of $\beta$ and JCR for $(\alpha, \beta)$, we define

$$
\varpi=\frac{W_{2}}{(m-1) W_{1}}=\frac{Y_{m}-Y_{1}}{(m-1) Y_{1}}
$$

and

$$
\xi=W_{1}+W_{2}=2 Y_{m} \text {. }
$$

It is clear that $\Phi \sim \frac{\chi^{2}(2 m-2)}{\chi^{2}(2)} \sim F(2 m-2,2)$ and $\xi \sim \chi^{2}(2 m)$ and also they are independent.

Theorem 3. Let $X_{U(i)} ; i=1,2, \ldots, m$ are first $i^{\text {th }}$ upper record values from a sequence of iid random variables following the $K U$ distribution with probability density function in (1.1). We consider $\phi\left(X_{U(1)}, X_{U(2)}, \ldots, X_{U(m)}, t\right)$ as a solution of $\beta$ for the equation

$$
\frac{\log \left(1-X_{U(m)}^{\beta}\right)-\log \left(1-X_{U(1)}^{\beta}\right)}{(m-1) \log \left(1-X_{U(1)}^{\beta}\right)}=t,
$$

Therefore, the $100(1-\gamma) \%$ ECI for $\beta$ is:

$$
\begin{aligned}
& {\left[\phi\left(X_{U(1)}, X_{U(2)}, \ldots, X_{U(m)}, F_{1-\gamma / 2}(2 m-2,2)\right),\right.} \\
& \left.\quad \phi\left(X_{U(1)}, X_{U(2)}, \ldots, X_{U(m)}, F_{\gamma / 2}(2 m-2,2)\right)\right]
\end{aligned}
$$

Proof of Theorem 3. We know that the

$$
\varpi=\frac{1}{(m-1)}\left(\frac{\log \left(1-X_{U(m)}^{\beta}\right)}{\log \left(1-X_{U(1)}^{\beta}\right)}-1\right),
$$

has a $F$ distribution with $2 m-2$ and $2 m$ degrees of freedom. Also, from Lemma 1 , $\varpi=t$ has a unique solution. So, analogous to Theorem 1 , the proof follows.

Theorem 4. Suppose we observe first $m$ upper record values, $X_{U(1)}, X_{U(2)}, \ldots$, $X_{U(m)}$ from the KU distribution, a $100(1-\gamma) \%$ JCR for $(\alpha, \beta)$ can be obtained by considering the following inequalities:

$$
\begin{gathered}
\phi\left(X_{U(1)}, X_{U(2)}, \ldots, X_{U(m)}, F_{(1+\sqrt{1-\gamma}) / 2}(2 m-2,2)\right)<\beta \\
<\phi\left(X_{U(1)}, X_{U(2)}, \ldots, X_{U(m)}, F_{(1-\sqrt{1-\gamma}) / 2}(2 m-2,2)\right) \\
-\frac{\chi_{(1+\sqrt{1-\gamma}) / 2}^{2}(2 m)}{2 \log \left(1-X_{U(1)}^{\beta}\right)}<\alpha<-\frac{\chi_{(1-\sqrt{1-\gamma}) / 2}^{2}(2 m)}{2 \log \left(1-X_{U(1)}^{\beta}\right)} .
\end{gathered}
$$


Proof of Theorem 4. Using the $\varpi$ and $\xi$ and further similar to the Theorem 2, we can conclude that

$$
\begin{gathered}
P\left[\phi\left(X_{U(1)}, X_{U(2)}, \ldots, X_{U(m)}, F_{(1+\sqrt{1-\gamma}) / 2}(2 m-2,2)\right)<\beta\right. \\
<\phi\left(X_{U(1)}, X_{U(2)}, \ldots, X_{U(m)}, F_{(1-\sqrt{1-\gamma}) / 2}(2 m-2,2)\right), \\
\left.-\frac{\chi_{(1+\sqrt{1-\gamma}) / 2}^{2}(2 m)}{2 \log \left(1-X_{U(m)}^{\beta}\right)}<\alpha<-\frac{\chi_{(1-\sqrt{1-\gamma} / 2}^{2}(2 m)}{2 \log \left(1-X_{U(m)}^{\beta}\right)}\right]=1-\gamma .
\end{gathered}
$$

\section{Simulations}

The goal of this simulation study is to examine the performance of the all proposed intervals or regions in terms of coverage probability with nominal level 0.95 . The coverage probability $(\mathrm{CP})$ is the probability that the interval or region contains the true parameters. All the computations were done using the $R$ programming language. We reported results from two simulation studies that we carried out. First, we generated a progressively censored sample from the KU distribution with arbitrarily true values $\alpha=2, \beta=1$ and different combination of $n$ and $m$. In our study we have used three different censoring schemes (C.S.), namely:

Scheme I: $R_{1}=n-m, R_{i}=0 ; i \neq 1$.

Scheme II: $R_{m}=n-m, R_{i}=0 ; i \neq m$.

Scheme III: $R_{1}=R_{4}=(n-m) / 2, R_{i}=0 ; i \neq 1,4$.

We computed the CPs for the ECI and JCR based on 10000 progressively censored samples generated from the $K U(2,1)$ distribution of size $m$. The results are presented in Table 1.

It is observed that from Table 1, the CPs of the ECI for and JCR for $(\alpha, \beta)$ are all close to the desired level of 0.95 . In the second simulation study, we obtained the CPs for the intervals and regions based on the upper record values. We simulated the upper record values $X_{U(i)} ; i=1,2, \ldots, m$ from the $\mathrm{KU}$ distribution with different values of $m$. We considered two cases for parameter values as:

Case 1: $\alpha=2, \beta=1$ and Case 2: $\alpha=2, \beta=2$.

The CPs of ECI and JCR for Case 1 (ECI1 and ECI1) and Case 2 (ECI2 and ECI2) are summarized in Table 2.

From Table 2, we observe that the CPs of the exact confidence interval and region are close to the desired level of 0.95 for different parameters and different values of $m$. 
TABLE 1. The coverage probability for ECI and JCR under different censoring schemes.

\begin{tabular}{ccccc}
\hline$n$ & $m$ & $C . S$. & $E C I$ & $J C R$ \\
\hline \multirow{2}{*}{20} & 16 & Scheme I & 0.9465 & 0.9414 \\
& 16 & Scheme II & 0.9488 & 0.9515 \\
& 16 & Scheme III & 0.9489 & 0.9509 \\
& & & & \\
30 & 20 & Scheme I & 0.9537 & 0.9522 \\
& 20 & Scheme II & 0.9531 & 0.9531 \\
& 20 & Scheme III & 0.9542 & 0.9493 \\
50 & 30 & & & \\
& 30 & Scheme I & 0.9489 & 0.9541 \\
& 30 & Scheme II & 0.9482 & 0.9522 \\
& & & 0.9542 & 0.9519 \\
& & & & \\
\hline
\end{tabular}

TABLE 2. The coverage probability for ECIs and JCRs under different upper record values.

\begin{tabular}{ccccc}
\hline$m$ & $E C I 1$ & $J C R I$ & $E C I 2$ & $J C R 2$ \\
\hline & & & & \\
5 & 0.9543 & 0.9495 & 0.9475 & 0.9544 \\
6 & 0.9475 & 0.9525 & 0.9486 & 0.9489 \\
7 & 0.9536 & 0.9530 & 0.9532 & 0.9527 \\
8 & 0.9518 & 0.9489 & 0.9529 & 0.9493 \\
9 & 0.9486 & 0.9493 & 0.9482 & 0.9530 \\
10 & 0.9538 & 0.9545 & 0.9485 & 0.9548 \\
11 & 0.9527 & 0.9528 & 0.9537 & 0.9494 \\
12 & 0.9514 & 0.9496 & 0.9529 & 0.9522 \\
& & & & \\
\hline
\end{tabular}

\section{REAl Data AnAlysis}

In this section, we first examine the suitability of the KU distribution to the two real data sets and then obtain the ECI for $\beta$ and JCR for $(\alpha, \beta)$ under PII censored sample and record values. 
Example 1. Brittle materials have many useful properties like stiffness, high strength retention at elevated temperatures, corrosion resistance associated with chemical inertness. Also, strength properties of brittle material are usually scattered; therefore, a statistical analysis is needed for the understanding of the mechanical characterization of this material. So, in this example, we consider the data of the strength property of extremely brittle solid ([3]). This dataset is reported to be recorded from the brittle glass. We have divided each data point by 100 and listed in Table 3.

TABLE 3. The strength data of the glass ceramic.

\begin{tabular}{c}
\hline $0.477,0.502,0.524,0.525,0.529,0.538,0.539,0.546,0.547$, \\
$0.549,0.555,0.564,0.575,0.590,0.600,0.611,0.614,0.624,0.627$, \\
$0.632,0.635,0.642,0.654,0.654,0.656,0.663,0.666,0.666$, \\
$0.668,0.672,0.675,0.676,0.680,0.684,0.696,0.704,0.707$, \\
$0.726,0.744$.
\end{tabular}

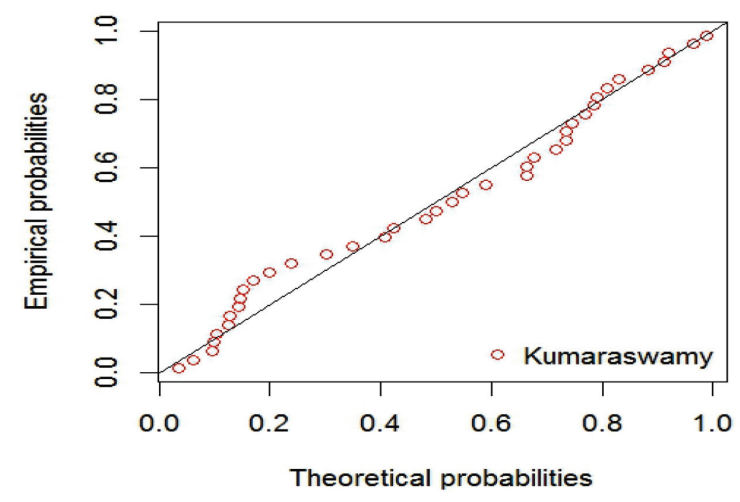

FIgURE 2. The P-P plot for the data of the strength property of brittle solid.

First we want to check whether the KU distribution fits the data or not and that we have applied only the complete data. The Kolmogorov-Smirnov (KS) distance between the empirical and the fitted distribution function and the associated p-value (in brackets) is $0.1125(0.7065)$. It clearly indicates that the KU provides a good fit to this data. We also present the P-P plot for the data in Figure 2. Figure 2 shows that the data do not deviate dramatically from the line. Therefore, the KU distribution provides a reasonable fit. Moreover, the strength properties of materials are commonly characterized by Weibull, log-normal and gamma strength distribution function. So, for comparison purposes, we present fitting of three other proposed distributions. The maximum likelihood estimates, Akaike's information criterion (AIC) and 
the associated second-order information criterion (AICc), Bayesian information criterion (BIC) and Kolmogorov-Smirnov (KS) statistic are presented in Table 4. From

TABLE 4. Estimated parameters, AICs, AICcs, BICs and K-S distances for different models.

\begin{tabular}{|c|c|c|c|c|c|}
\hline Models & $M L E$ & $A I C$ & $A I C c$ & $B I C$ & $K S$ \\
\hline Kumaraswamy & $\begin{array}{l}\alpha=104.459 \\
\beta=10.7564\end{array}$ & -96.81415 & -96.48082 & -93.48703 & 0.112569 \\
\hline Weibull & $\begin{array}{c}\text { shape }=10.8193 \\
\text { scale }=0.64867\end{array}$ & -96.79048 & -96.45715 & -93.46336 & 0.113163 \\
\hline Log-normal & $\begin{array}{l}\text { meanlog }=1.000 \\
\text { sdlog }=0.000061\end{array}$ & -94.33432 & -94.00099 & -91.00719 & 0.144718 \\
\hline Gamma & $\begin{array}{c}\text { shape }=81.8871 \\
\text { scale }=0.00756\end{array}$ & -94.88559 & -94.55226 & -91.55847 & 0.141601 \\
\hline
\end{tabular}

Table 4, the KU distribution gives a better fit than other proposed distribution for this dataset. To use this data set under PII censoring schemes, we have created the PII censoring schemes as:

Censoring Scheme: $n=39, m=17, R_{1}=R_{2}=\ldots=R_{11}=2, R_{i}=0 ; i=1,2, \ldots, 11$. For computing the ECI for $\beta$ under complete $\left(x^{\text {complete }}\right)$ and PII censored $\left(x^{P I I}\right)$ sample, we need the following percentiles;

$$
\begin{aligned}
& F_{0.975}(2(39)-2,2)=0.25814, F_{0.025}(2(39)-2,2)=39.4847, \\
& F_{0.975}(2(17)-2,2)=0.24103, F_{0.025}(2(17)-2,2)=39.4667 .
\end{aligned}
$$

Based on the above percentiles, the lower and upper bound for the $95 \%$ ECI of $\beta$ under $x^{\text {complete }}$ and $x^{P I I}$ can be obtained as:

$$
\begin{aligned}
& \beta_{L}=\varphi\left(x^{\text {complete }}, 0.25814\right)=7.96316 \\
& \beta_{U}=\varphi\left(x^{\text {complete }}, 39.4847\right)=21.6431
\end{aligned}
$$

and

$$
\begin{gathered}
\beta_{L}=\varphi\left(x^{P I I}, R_{1}=R_{2}=\ldots=R_{11}=2,0.24103\right)=5.8566 \\
\beta_{U}=\varphi\left(x^{P I I}, R_{1}=R_{2}=\ldots=R_{11}=2,39.4667\right)=20.1307 .
\end{gathered}
$$

respectively. Therefore under $x^{\text {complete }}$ and $x^{P I I}$ the $95 \% \mathrm{ECI}$ of $\beta$ are:

$$
7.96316<\beta<21.6431 \text {, }
$$

and

$$
5.8566<\beta<20.1307 .
$$


respectively. Also, to obtain the $95 \%$ JCR for $(\alpha, \beta)$, we present the following percentiles:

$$
\begin{gathered}
F_{0.9873}(2(39)-2,2)=0.2161, F_{0.0127}(2(39)-2,2)=78.2259, \\
F_{0.9873}(2(17)-2,2)=0.1992, F_{0.0127}(2(17)-2,2)=78.2079, \\
\chi_{0.0127}^{2}(78)=108.5374, \chi_{0.9873}^{2}(78)=52.7792, \\
\chi_{0.0127}^{2}(34)=55.0243, \chi_{0.9873}^{2}(34)=18.2764 .
\end{gathered}
$$

So, for $x^{\text {complete }}$ and $x^{P I I}$, we have

$$
\beta_{L}=\varphi\left(x^{\text {complete }}, 0.2161\right)=7.4648 \& \beta_{U}=\varphi\left(x^{\text {complete }}, 78.2259\right)=23.4040,
$$

and

$$
\begin{gathered}
\beta_{L}=\varphi\left(x^{P I I}, R_{1}=R_{2}=\ldots=R_{11}=2,0.1992\right)=5.3276 \\
\& \quad \beta_{U}=\varphi\left(x^{P I I}, R_{1}=R_{2}=\ldots=R_{11}=2,78.2079\right)=21.912 .
\end{gathered}
$$

respectively. So, the $95 \%$ JCR for $(\alpha, \beta)$ under $x^{\text {complete }}$ and $x^{P I I}$ can be written as:

$$
\left\{\begin{array}{c}
7.4648<\beta<23.4040 \\
-\frac{52.7792}{2 \sum_{i=1}^{39} \log \left(1-X_{i: 39}^{\beta}\right)}<\alpha<-\frac{108.5374}{2 \sum_{i=1}^{39} \log \left(1-X_{i: 39}^{\beta}\right)},
\end{array}\right.
$$

and

$$
\left\{\begin{array}{c}
5.3276<\beta<21.912 \\
-\frac{18.2764}{2 \sum_{i=1}^{17}\left(R_{i}+1\right) \log \left(1-X_{i: 17: 39}^{\beta}\right)}<\alpha<-\frac{55.0243}{2 \sum_{i=1}^{17}\left(R_{i}+1\right) \log \left(1-X_{i: 17: 39}^{\beta}\right)},
\end{array}\right.
$$

respectively. Also, the confidence region for $(\alpha, \beta)$ under the complete and PII cen-

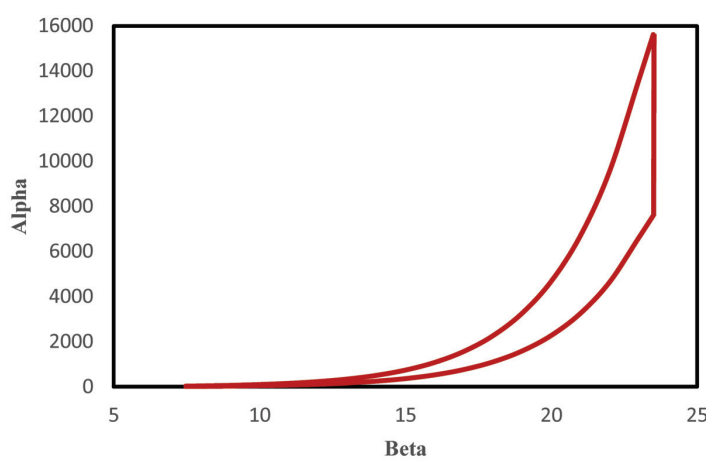

FIGURE 3. Joint confidence regions for $(\alpha, \beta)$ based on complete data in Example 1.

sored samples are displayed in Figures 3 and 4 respectively. It is easy see that the region is large when $\beta$ is large. 


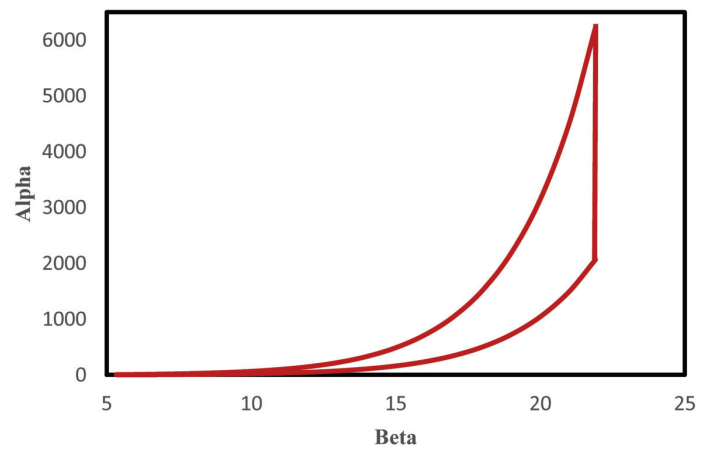

FIGURE 4. Joint confidence regions for $(\alpha, \beta)$ based on censored data in Example 1.

Example 2. The wind energy is an alternative clean energy source compared with the fossil fuels that pollute the lower layer of the atmosphere. As the wind speed is random phenomenon, the statistical distribution is required for the wind speed. For this reason, we consider the daily average wind speeds from 2001-2006 for Manjil city (http://www.chaharmahalmet.ir/stat/archive/iran/gil/MANJIL/36.asp). The data after dividing by 100 are given in Table 5 .

TABLE 5. The data of the daily average wind speeds.

$0.063,0.081,0.120,0.125,0.169,0.227,0.221,0.207,0.172,0.119,0.065$, $0.067,0.136,0.064,0.074,0.096,0.139,0.169,0.211,0.211,0.234,0.148$, $0.106,0.052,0.059,0.130,0.052,0.059,0.117,0.123,0.130,0.214,0.219$, $0.166,0.163,0.085,0.061,0.040,0.120,0.042,0.060,0.113,0.10,0.111$, $0.175,0.192,0.178,0.152,0.095,0.043,0.048,0.110,0.0320 .0490 .084$, $0.110,0.101,0.194,0.197,0.185,0.164,0.100,0.048,0.044,0.109,0.046$, $0.042,0.088,0.090,0.127,0.179,0.187,0.125,0.143,0.082,0.055,0.039,0.100$.

Here, we restricted ourselves to fitting the KU distribution to this dataset and determining the adequacy of KU distribution using the Kolmogorov-Smirnov statistic between the fitted and the empirical distributions. This statistic and the associated $\mathrm{p}$ value are 0.0899 and 0.5528 . Therefore, based on the p-value we can say that the KU distribution fits to this data. Now, we extract a sample of upper record values from this data set as: $\mathbf{0 . 0 6 3}, \mathbf{0 . 0 8 1}, \mathbf{0 . 1 2 0}, \mathbf{0 . 1 2 5}, \mathbf{0 . 1 6 9}, \mathbf{0 . 2 2 7}$ and 0.234 . To demonstrate interval methods, we consider the following percentiles:

$$
\begin{gathered}
F_{0.025}(12,2)=39.4146, F_{0.975}(12,2)=0.1962, F_{0.9873}(12,2)=0.1557, \\
F_{0.0127}(12,2)=78.1558, \chi_{0.0127}^{2}(14)=28.3704, \chi_{0.9873}^{2}(14)=4.889 .
\end{gathered}
$$


Based on upper record values, we have:

and

$$
\phi\left(X_{U(1)}, X_{U(2)}, \ldots, X_{U(7)}, 0.1962\right)=0.4569
$$

$$
\phi\left(X_{U(1)}, X_{U(2)}, \ldots, X_{U(7)}, 39.4146\right)=4.1678 .
$$

So by using Theorem 3 , the $95 \%$ ECI of $\beta$ is $(0.4569,4.1678)$. Also using Theorem 4 ,

and

$$
\phi\left(X_{U(1)}, X_{U(2)}, \ldots, X_{U(7)}, 0.1557\right)=0.35426
$$

$$
\phi\left(X_{U(1)}, X_{U(2)}, \ldots, X_{U(7)}, 78.1558\right)=4.68839 .
$$

Therefore, the $95 \%$ JCR for $(\alpha, \beta)$ is:

$$
\left\{\begin{array}{c}
0.35426<\beta<4.68839 \\
-\frac{4.889}{2 \log \left(1-X_{U(m)}^{\beta}\right)}<\alpha<-\frac{28.3704}{2 \log \left(1-X_{U(m)}^{\beta}\right)} .
\end{array}\right.
$$

Figure 5 shows the $95 \%$ confidence region for $(\alpha, \beta)$. It is easy to see that the region is large when $\beta$ is large.

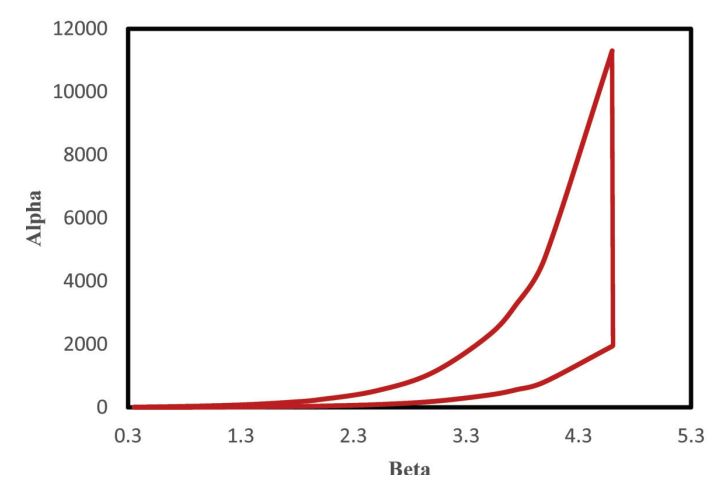

FIGURE 5. Joint confidence regions for $(\alpha, \beta)$ based on record values in Example 2.

\section{CONCLUSION}

The subject of progressively censoring and the record values have been received considerable attention in the past few years. The KU distribution and its new family can be applied in different sciences because of various forms of its hazard function. We computed the ECI of $\beta$ and the JCR for $(\alpha, \beta)$ under the PII censored sample and upper record values using different pivotal quantities. The simulation study shows that the proposed intervals work well and the CPs of the intervals and regions are close to the desired level of 0.95 . Finally, two real data sets contain the strength 
of glass ceramic and the daily average wind speeds are analyzed to illustrate our methods.

\section{REFERENCES}

[1] T. A. Abushal and A. A. Soliman, "Estimating the Pareto parameters under progressive censoring data for constant-partially accelerated life tests," Journal of Statistical Computation and Simulation, vol. 85, no. 5, pp. 917-934, 2015, doi: 10.1080/00949655.2013.853768.

[2] B. C. Arnold, N. Balakrishnan, and H. N. Nagaraja, "Records," John Wiley, New York, 1998.

[3] B. Basu, D. Tiwari, D. Kundu, and R. Prasad, "Is Weibull distribution the most appropriate statistical strength distribution for brittle materials?" Ceramics International, vol. 35, no. 1, pp. 237-246, 2009, doi: 10.1016/j.ceramint.2007.10.003.

[4] A. Childs, B. Chandrasekar, N. Balakrishnan, and D. Kundu, "Exact likelihood inference based on Type-I and Type-II hybrid censored samples from the exponential distribution," Annals of the Institute of Statistical Mathematics, vol. 55, no. 2, pp. 319-330, 2003, doi: 10.1007/BF02530502.

[5] S. Dey, T. Dey, and D. J. Luckett, "Statistical inference for the generalized inverted exponential distribution based on upper record values," Mathematics and Computers in Simulation, vol. 120, pp. 64-78, 2016, doi: 10.1016/j.matcom.2015.06.012.

[6] S. Dey, J. Mazucheli, and S. Nadarajah, "Kumaraswamy distribution: different methods of estimation," Computational and Applied Mathematics, vol. 37, no. 2, pp. 2094-2111, 2018, doi: 10.1007/s40314-017-0441-1.

[7] I. Elbatal and M. Elgarhy, "Statistical properties of Kumaraswamy quasi Lindley distribution," International Journal of Mathematics Trends and Technology, vol. 4, no. 10, pp. 237-246, 2013.

[8] M. Garg, "On distribution of order statistics from Kumaraswamy distribution," Kyungpook mathematical journal, vol. 48, no. 3, pp. 411-417, 2008, doi: 10.5666/KMJ.2008.48.3.411.

[9] K. Huang and J. Mi, "Inference about Weibull distribution using upper record values," International Journal of Reliability, Quality and Safety Engineering, vol. 22, no. 04, p. 1550016, 2015, doi: 10.1142/S0218539315500163.

[10] M. Jones, "Kumaraswamy distribution: A beta-type distribution with some tractability advantages," Statistical Methodology, vol. 6, no. 1, pp. 70-81, 2009, doi: 10.1016/j.stamet.2008.04.001.

[11] P. Kumaraswamy, "A generalized probability density function for double-bounded random processes," Journal of Hydrology, vol. 46, no. 1-2, pp. 79-88, 1980, doi: 10.1016/00221694(80)90036-0.

[12] D. Kundu and H. Howlader, "Bayesian inference and prediction of the inverse Weibull distribution for Type-II censored data," Computational Statistics \& Data Analysis, vol. 54, no. 6, pp. 15471558, 2010, doi: 10.1016/j.csda.2010.01.003.

[13] A. J. Lemonte, "Improved point estimation for the Kumaraswamy distribution," Journal of Statistical Computation and Simulation, vol. 81, no. 12, pp. 1971-1982, 2011, doi: 10.1080/00949655.2010.511621.

[14] C. T. Lin and N. Balakrishnan, "Asymptotic properties of maximum likelihood estimators based on progressive Type-II censoring," Metrika, vol. 74, no. 3, pp. 349-360, 2011, doi: 10.1007/s00184010-0306-8.

[15] P. A. Mitnik, "New properties of the Kumaraswamy distribution," Communications in StatisticsTheory and Methods, vol. 42, no. 5, pp. 741-755, 2013, doi: 10.1080/03610926.2011.581782.

[16] M. Nadar, A. Papadopoulos, and F. Kizılaslan, "Statistical analysis for Kumaraswamy distribution based on record data," Statistical Papers, vol. 54, no. 2, pp. 355-369, 2013, doi: 10.1007/s00362012-0432-7. 
[17] H. Panahi, "Exact confidence interval for the generalized inverted exponential distribution with progressively censored data," Malaysian Journal of Mathematical Sciences, vol. 11, no. 3, pp. 331-345, 2017.

[18] H. Panahi, "Estimation of the Burr Type III distribution with application in unified hybrid censored sample of fracture toughness," Journal of applied Statistics, vol. 44, no. 14, pp. 2575-2592, 2017, doi: 10.1080/02664763.2016.1258549.

[19] H. Panahi and A. Sayyareh, "Estimation and prediction for a unified hybrid-censored Burr Type XII distribution," Journal of Statistical Computation and Simulation, vol. 86, no. 1, pp. 55-73, 2016, doi: 10.1080/00949655.2014.993985.

[20] B. Pradhan and D. Kundu, "On progressively censored generalized exponential distribution," Test, vol. 18, no. 3, pp. 497-515, 2009, doi: 10.1007/s11749-008-0110-1.

[21] M. Z. Raqab, "Inferences for generalized exponential distribution based on record statistics," Journal of statistical planning and inference, vol. 104, no. 2, pp. 339-350, 2002, doi: 10.1016/S0378-3758(01)00246-4.

[22] H. Saulo, J. Leão, and M. Bourguignon, "The Kumaraswamy Birnbaum-Saunders distribution," Journal of Statistical Theory and Practice, vol. 6, no. 4, pp. 745-759, 2012, doi: 10.1080/15598608.2012.719814.

[23] P. Y. Thomas and J. Paul, "On generalized lower (k) record values from the Fréchet distribution," Journal of the Japan Statistical Society, vol. 44, no. 2, pp. 157-178, 2014, doi: $10.14490 /$ jjss.44.157.

[24] S. F. Wu, C. C. Wu, Y. L. Chen, Y. R. Yu, and Y. P. Lin, "Interval estimation of a two-parameter Burr-XII distribution under progressive censoring," Statistics, vol. 44, no. 1, pp. 77-88, 2010, doi: 10.1080/02331880902757922.

[25] L. Xiaohu, H. Yanyan, and Z. Xueyan, "The Kumaraswamy binomial distribution," Chinese Journal of Applied Probability and Statistics, vol. 27, no. 5, pp. 511-521, 2011.

Author's address

Hanieh Panahi

Department of Mathematics and Statistics, Lahijan branch, Islamic Azad University, Lahijan, Iran

E-mail address: panahi@liau.ac.ir 